\title{
EXPLICAR Y CONTRASTAR
}

\author{
SANTIAgo GinNOBILI \\ Universidad de Buenos Aires \\ Universidad Nacional de Quilmes \\ CONICET \\ santi75@gmail.com \\ Christí́n CARMAN \\ Universidad Nacional de Quilmes \\ CONICET \\ ccarman@gmail.com
}

RESUMEN: Usualmente se ha asumido que una única distinción puede dar cuenta del papel que cumplen los conceptos en una teoría respecto de la contrastación y respecto de la explicación. Intentaremos mostrar que esta asunción es incorrecta. Por una parte, no hay razones para considerar que esta coincidencia deba darse y, por otra, como pretenderemos probar a partir de varios ejemplos, de hecho, no se da. La base de contrastación de una teoría no tiene por qué coincidir con el explanandum de la teoría. Para defender este punto asumiremos el estructuralismo metateórico, y se extraerán consecuencias para la concepción metateórica presupuesta.

PALABRAS CLAVE: explicación, contrastación, T-teoricidad, estructuralismo metateórico, distinción teórico observacional

SUMMARY: It is usually held that one distinction can account for the role that concepts play in a theory regarding both test and explanation. We will demonstrate that this assumption is incorrect. On the one hand, there is no reason to think that this coincidence should exist. On the other, this is not the case, as we will show analysing several examples. The testing basis of a theory does not have to coincide with the explanandum of the theory. To defend this point we will endorse the metatheoretical structuralism. In addition, we will consider some repercussions that this discussion has for the assumed metatheoretical framework.

KEY WORDS: explanation, test, T-theoreticity, metatheoretical structuralism, theoretical observational distinction

Desde el inicio mismo de su etapa profesionalizada, existe, en la filosofía de la ciencia, la idea intuitiva según la cual es posible separar los términos o conceptos de una teoría en dos clases según el papel que cumplen en ella. Por citar algunos ejemplos, el papel de la fuerza en la mecánica clásica es distinto al de la aceleración; en la astronomía ptolemaica, aquel de los epiciclos y deferentes difiere del de la posición de los planetas en el cielo; en la genética, el papel que cumplen los genes difiere del que cumplen los parecidos fenotípicos entre los progenitores y su descendencia, etc. En los casos citados, los primeros desempeñan un papel distinto al de los segundos en 
dos aspectos: en primer lugar, por su papel explicativo - las teorías utilizan dichos conceptos para dar cuenta del comportamiento de las entidades referidas por los segundos-y, en segundo, por su papel en la contrastación - la determinación de los segundos permite contrastar las afirmaciones que la teoría hace con la ayuda de los primeros-.

Por supuesto que el hecho de que intuitivamente la distinción sea relativamente clara y la aplicación en la mayoría de los casos no sea problemática no implica que una elucidación adecuada del criterio que se usa para la distinción en cuestión sea sencilla. Y como desde el siglo XX la historia de la filosofía de la ciencia muestra, no hay cuestión que haya sido tan discutida como ésta.

Considerando teorías que enriquecen conceptualmente su "base empírica", " generalmente se ha asumido que los conceptos que en una teoría categorizan el fenómeno que dicha teoría quiere explicar son los mismos que por ser pasibles de ser aplicados con independencia de esta teoría, permiten contrastarla, por una parte. Por la otra, que los conceptos que la teoría propone para explicar sus explanan$d a$, son aquellos que no pueden determinarse con independencia de la teoría, y que, en consecuencia, no permiten contrastarla. En este sentido, uno y el mismo criterio permitiría determinar el papel que los conceptos desempeñan tanto respecto de la explicación como respecto de la contrastación. A estas alturas no podemos ser más precisos en la enunciación de este punto, puesto que su correcta enunciación implica proponer nuevas distinciones que desarrollaremos a lo largo del presente trabajo.

Defenderemos que no hay razones conceptuales para sustentar esta coincidencia, es decir, que el papel que un concepto desempeña con respecto a la contrastación permite determinar su papel con respecto a la explicación. Respaldaremos esta hipótesis con el análisis de algunos ejemplos de teorías científicas.

El estructuralismo metateórico (Balzer, Moulines, y Sneed 1987) brinda herramientas conceptuales para poder presentar esta problemática de manera clara y precisa, en consecuencia, utilizaremos las herramientas metateóricas que este enfoque brinda. Que las distinciones entre conceptos según su papel explicativo y según su papel en la contrastación no coincidan ni intensional ni extensionalmente

\footnotetext{
${ }^{1}$ Tanto en el enfoque clásico como en el estructuralismo metateórico, se acepta que algunas teorías no incluyan nuevos conceptos (el ejemplo paradigmático suele ser la cinemática galileana). Este trabajo versará todo el tiempo sobre las teorías que sí los incluyen (en sentidos que iremos aclarando a lo largo del mismo).
} 
- la hipótesis de este trabajo - es un resultado significativo para la filosofía de la ciencia en general, y, además, tiene consecuencias para el estructuralismo metateórico en particular, como pretendemos mostrar.

\section{Explicación y contrastación en la concepción heredada}

En la concepción heredada se pretendía capturar la distinción intuitiva que nos ocupa a través de la observabilidad. Los términos teóricos (entendidos como no observacionales) constituirían los elementos propios de una teoría - aquellos que la teoría utiliza para explicar- y los observacionales los que no le son propios - aquellos que garantizan la contrastación independiente de la teoría-. La presentación paradigmática de esta posición puede encontrarse en "El carácter metodológico de los términos teóricos" (1956). Allí, Carnap afirma:

En metodología de las ciencias se acostumbra, por razones de utilidad, dividir el lenguaje científico en dos partes, el lenguaje de observación y el lenguaje teórico. El primero usa términos que designan propiedades y relaciones observables, para los efectos de la descripción de objetos o hechos igualmente observables, el lenguaje teórico, a su vez, contiene términos que pueden referirse a hechos inobservables o a aspectos o rasgos inobservables de los hechos [...] uno de los temas principales será el problema de un criterio de significación para el lenguaje teórico; es decir, las condiciones exactas que deberán darse en los términos y proposiciones del lenguaje teórico para cumplir una función efectiva en la explicación y predicción de los hechos observables y así poder aceptarlos como empíricamente significativos. (1956, p. 38, las cursivas son nuestras.)

Había, al menos, dos razones claras para trazar dicha distinción: a) los términos observacionales tenían garantizada su legitimidad referencial por referirse a objetos, propiedades o relaciones observables; los términos teóricos, por su parte, debían adquirir esa garantía a través de una interpretación parcial a partir de su vinculación con términos observacionales (mediante principios puente o reglas de correspondencia); b) los términos observacionales proveían una base lo suficientemente segura, relativamente objetiva, y en consecuencia independiente, para contrastar los enunciados teóricos.

Lo relevante para nuestros propósitos es que con uno y el mismo criterio se pretendía dar cuenta de ambos papeles, con respecto a la explicación y a la contrastación. 
Por supuesto, es posible entender por qué se consideraba que la distinción basada en la observabilidad podría cumplir ambas tareas. Pues, en una primera mirada, en teorías simples, pero que proponen conceptos nuevos, ${ }^{2}$ parece que tales conceptos suelen ser no observacionales y las predicciones que se hacen suelen utilizar exclusivamente conceptos observacionales. Es decir, en estos casos se contrastaría a la teoría con enunciados empíricos y se querría explicar la conducta de entidades observables. Un ejemplo podría constituirlo (en una primera instancia) la teoría de Mendel. Para explicar las frecuencias encontradas de rasgos en las cruzas de las variedades puras, Mendel propuso elementos inobservables que determinarían el rasgo en los organismos en cuestión (1865). Los rasgos a considerar (p.ej. color y rugosidad de las semillas) serían directamente observables, mientras que los elementos postulados serían inobservables. La posesión de ciertos elementos inobservables explicaría la posesión de rasgos en un organismo particular, y, por otra parte, la vinculación de esos elementos inobservables con rasgos nos permitiría contrastar las leyes que Mendel propuso.

\section{Críticas a la distinción de la concepción clásica}

Como es bien conocido, esta distinción recibió fuertes críticas, unas orientadas a subrayar que los criterios propuestos hasta el momento no lograban separar satisfactoriamente los términos que intuitivamente debían caer de un lado o del otro de la distinción (Achinstein 1963, 1965, 1968; Hempel 1958, 1966, 1970; Putnam 1962; Suppe 1972) y otras, más radicales, apuntaban a mostrar la invalidez de la misma razón que motivó la distinción ya que la tesis de la carga teórica de la observación señalaría que los términos teóricos afectan el significado de los observacionales y que, justamente por esto, los observacionales no pueden ser una base empírica fiable para contrastar teorías (Hanson 1958; Kuhn 1962; Popper 1962).

A principios de los años sesenta algunos autores ya empezaban a intuir que no era lícito identificar lo teórico con lo no observacional (Putnam 1962). Mientras el carácter observacional o no observacional de un término no dependiera de la teoría en la que ocurre, su carácter de teórico sí lo haría. Así, Achinstein afirma que:

uno siempre debe especificar la teoría con respecto a la cual un término dado está o no está "cargado de teoría". Y parecería que un término podría recibir esta clasificación respecto de una teoría, pero no respecto

${ }^{2}$ Que no sean meras definiciones en términos de conceptos observacionales. 
de otra, aun cuando el término ocurra en ambas. Puesto que en una teoría su significado no podría entenderse a menos que los principios de la teoría sean conocidos, mientras que éste no necesariamente sería el caso para la otra teoría (o al menos podría haber diferencias de grado significativas). (1965, p. 200; la traducción es nuestra.)

Varios autores denunciaron que la distinción teórico observacional suponía la confusión de dos dicotomías, en algún sentido relacionadas, pero diferentes: la dicotomía entre lo observacional y lo no observacional y la dicotomía entre lo teórico y lo no-teórico (BarHillel 1970, pp. 267-269; Lewis 1970; Ruse 1973, cap. 2.2). Un buen ejemplo de esta nueva diferenciación, otra vez, proviene de la genética clásica. Aplicando la distinción teórico observacional, en genética clásica podríamos vernos tentados a considerar los rasgos fenotípicos como observacionales y los genes como teóricos. Sin embargo, aunque en ciertos casos los rasgos son observables, no siempre es así. Por ejemplo, el factor sanguíneo es un rasgo que se hereda según las leyes de la genética clásica. Difícilmente alguien podría considerarlo observable (en el sentido de la concepción heredada) puesto que tiene que ver con la presencia o no de una proteína particular en la membrana de los glóbulos rojos, que se determina por medio de procedimientos indirectos a través de reactivos. En consecuencia, algunas de las entidades que pertenecen a la extensión del concepto de rasgo no serían observables aunque el concepto de rasgo, en principio, como habíamos visto, sería no teórico en esta teoría. El concepto de rasgo no sería sencillamente catalogable como observacional, puesto que bajo su extensión caerían tanto entidades observables como inobservables.

Una de las propuestas positivas más interesantes que separa a la teoricidad de la observabilidad es la distinción que Hempel hace entre los conceptos que se encontraban disponibles con anterioridad a una determinada teoría y los que se proponen con dicha teoría (1970). Según Hempel, en una teoría podría distinguirse entre aquellos conceptos que la teoría propone para dar cuenta de aquellos fenómenos de los cuales quiere dar cuenta, de aquellos conceptos que permiten describir el fenómeno a explicar, que se encontrarían disponibles antes de la postulación de la teoría, y que serían comprensibles independientemente de ésta. Sin embargo, como el mismo Hempel sostiene, para aplicar el criterio es necesario determinar si un concepto que aparece en una teoría es el mismo que el que aparece en una teoría anterior o no, esto es, se requiere una noción de igualdad de significado (1970). Es decir, la aplicación del criterio presupone nociones semánticas. Esto, por supuesto, invita a pensar 
que las que, a final de cuentas, permitirán reconstruir claramente la distinción buscada serán aquellas nociones semánticas presupuestas en el criterio historiográfico hempeliano.

Para nuestros fines, lo importante es destacar que, aunque Hempel abandona la distinción basada en la observabilidad, sigue sosteniendo, explícitamente, la identificación de aquello que permite contrastar la teoría y aquello que la teoría permite explicar. Los conceptos que una teoría propone permiten explicar aquellos fenómenos descriptos a través de conceptos que están disponibles con anterioridad a esta teoría $(1970, \S 1-2)$.

De nuevo, se espera que sea así en muchos casos (al menos bajo una primera mirada), pues el fenómeno a explicar normalmente se encuentra conceptualizado previamente (en sentido histórico) a la teoría que lo explica, y se espera además que la determinación de los conceptos comprensibles independientemente de la teoría brinde una base de contrastación independiente. De hecho, esta distinción permite mostrar que la tesis de la carga teórica no tiene la consecuencia indeseable de que la base empírica de una teoría pierda su independencia. En este caso, la base "empírica" (entre comillas ahora porque no necesariamente estaría formada por fenómenos observables, o por enunciados que describen fenómenos observables) no es absoluta y está cargada de teoría, pero no de la teoría que se quiere contrastar. La contrastación no se realizaría con una base empírica absoluta, pero si de manera independiente de la teoría en cuestión. Siguiendo con nuestro ejemplo, la genética clásica habría propuesto el concepto de gen para dar cuenta del modo en que se heredan los rasgos. $\mathrm{Y}$ es a través de la determinación independiente de la teoría de los rasgos que poseen los organismos que se contrastan las leyes de la genética clásica, aunque éstos no sean en todos los casos observables (como ocurre con el factor sanguíneo).

\section{La propuesta de Sneed}

Sneed (1971), en el mismo espíritu que los autores citados en la sección anterior propone una distinción superadora que se incorporará posteriormente al marco brindado por el estructuralismo metateórico:

El estructuralismo rechaza la distinción "teórico/observacional" por ambigua. Esta distinción esconde en realidad dos: "observable/inobservable" de un lado, y "no teórico/teórico" de otro. Ambas distinciones no coinciden intensionalmente ni extensionalmente. [...] Un término no es teórico o no teórico sin más, sino relativamente a una teoría dada. 
Por eso no se debe hablar tanto de teoricidad cuanto de T-teoricidad, teoricidad relativamente a la teoría T. La idea que hay detrás es, expresada en términos modeloteóricos, similar a la distinción que vimos del último Hempel entre vocabulario-antecedente y vocabulario propio [...]. La idea es que un concepto es T-teórico si es un concepto propio de la teoría T, "introducido" por ella, y es T-no teórico si es un concepto disponible previamente a T. La cuestión es precisar esta intuición. [...] Pues bien, si un concepto es T-no teórico, si es "anterior" a $\mathrm{T}$, entonces tendrá al menos algunos procedimientos de determinación independientes de T; en cambio si es T-teórico, si es propio de T, su determinación depende siempre de T. (Díez y Moulines 1997, pp. 354355)

Así, el criterio de distinción entre lo T-teórico y lo T-no teórico está vinculado ahora con los procedimientos de determinación de los conceptos, y ya no con la precedencia temporal. La distinción sería sistemática y no historiográfica. En conceptos cuantitativos, la determinación consiste justamente en la determinación de un valor numérico. Si para determinarlo es necesario apelar a las leyes propias de la teoría, será un concepto teórico para esa teoría, mientras que, si puede determinarse su valor sin apelar a la teoría, significa que no es teórico en esa teoría. Así, por ejemplo, si para calcular la fuerza que actúa sobre un cuerpo es necesario aplicar las leyes de la mecánica de Newton, y no es necesario hacerlo para calcular su aceleración, entonces la primera será teórica para la mecánica clásica de Newton, mientras que la segunda no lo será. ${ }^{3}$ En esta propuesta, el criterio de la distinción no está vinculado con la observabilidad, sino con la contrastabilidad. Aun cuando los conceptos en cuestión no sean observacionales, e incluso aun cuando estén cargados de teoría, puesto que la teoría de la que están cargados no es la misma que la que se desea contrastar, poseemos una base "empírica" independiente y, por lo tanto, no caemos tampoco en ninguna circularidad:

${ }^{3}$ Estrictamente en el estructuralismo metateórico se distingue entre dos criterios de T-teoricidad (Balzer et al. 1987, pp. 47-78). Lo que acabamos de presentar es el criterio informal de T-teoricidad. En el criterio formal de T-teoricidad se considera que un concepto es T-no teórico si no existen métodos de determinación para dicho concepto en T. Y es T-teórico si es posible determinar el concepto a partir de T. En este trabajo consideraremos sólo el criterio informal, porque es el que se relaciona de manera más directa con la contrastabilidad. Basta para que una teoría sea contrastable independientemente del hecho de que sea posible determinar alguno de sus conceptos desde otras teorías. Por lo tanto, siempre que hablemos en este trabajo de la T-teoricidad, nos estaremos refiriendo al criterio informal. 
La distinción entre conceptos específicos para una teoría dada T y conceptos no específicos para $\mathrm{T}$ ha desempeñado un papel importante en el desarrollo de la filosofía de la ciencia en los últimos cuarenta años. La distinción está estrechamente relacionada con la cuestión de cómo podemos proveer una "base empírica" para una teoría, e.e., un marco de conceptos por medio de los cuales la teoría pueda ser controlada o por medio de los cuales podamos averiguar cuándo la teoría funciona correctamente y cuándo no. La "base empírica" de una teoría dada $\mathrm{T}$ debería incluir aquellos conceptos que, en un sentido aún por precisar, no son específicos para T. Los otros conceptos de T pertenecerán a la "superestructura" teórica de T. (Balzer et al. 1987, p. 48)

Gracias a la distinción de T-teoricidad puede presentarse con claridad lo que Lorenzano ha llamado "base empírica global de una teoría": "constituida por el conjunto Mpp de los modelos parciales, que describen, mediante conceptos no-teóricos o "empíricos" relativamente a la teoría en cuestión, los sistemas posibles a los que es concebible aplicar (globalmente) dicha teoría" (2012, p. 85).

La noción de "base empírica global" sería análoga a la noción de "base empírica" utilizada en la concepción clásica, en el sentido de que es un concepto relativo a una teoría en general y no a sus contrastaciones particulares. Y en el caso de la noción estructuralista no necesariamente estaría formada, como veíamos, por fenómenos o sistemas observables, y no sería absoluta, sino relativa a una teoría. La base empírica global, que técnicamente se obtiene al recortar de los modelos potenciales de una teoría sus componentes teóricos, permitiría mostrar con claridad la base de contrastación de la teoría. Esto es así porque todos los conceptos que aparecen en la base empírica global serían no teóricos, y, en consecuencia, determinables independientemente de la teoría. La noción de "base empírica global" Lorenzano la introduce en contraste con la "base empírica local". La base empírica local sería lo que funciona como base empírica en una contrastación particular, que puede incluir conceptos teóricos. Pues en aplicaciones particulares puede ocurrir que se reciban como dados valores de términos que, aunque teóricos, han sido determinados en aplicaciones anteriores de esa misma teoría.

Lo que nos interesa señalar es que en esta concepción sigue considerándose (tal vez como herencia del enfoque clásico) la idea de que un único criterio permite elucidar tanto el papel de los conceptos con respecto a la contrastabilidad, como el papel de los conceptos con respecto a la explicatividad. P.ej., en el texto ya citado Lorenzano afirma: 
la distinción entre "base empírica de contrastación global" y "base empírica de contrastación local" [...] no desdibuja la diferenciación que puede hacerse en general, o "globalmente", en una teoría, y debiera tenerse presente, entre los "hechos a ser explicados" y "la construcción teórica" (en la terminología de Ramsey) o entre los sistemas "empíricos" a ser explicados (las aplicaciones intencionales formuladas en el vocabulario T-no-teórico y representadas mediante modelos parciales Mpp en su caracterización estándar) y las extensiones T-teóricas, leyes y condiciones de ligadura (en terminología estructuralista). (2012, pp. 8687)

Así se considera que la distinción que permite identificar aquellos conceptos que por ser determinables con independencia de la teoría en cuestión permiten contrastarla, también permite distinguir entre los conceptos con los que se describen los fenómenos a explicar en el marco de una teoría dada, de aquellos conceptos que permiten dar cuenta bajo esa teoría de tales fenómenos. Es decir, que la base empírica global, formada por conceptos no teóricos, coincide con el que podríamos llamar "explanandum global" de la teoría, asumiéndose aquí también cierta identidad entre explicación y predicción. ${ }^{4}$

${ }^{4}$ La idea de "explanandum global" y "explanans global" permite hablar del papel explicativo o no explicativo de los conceptos en una teoría (en un sentido que se aclarará posteriormente). No hay que confundir esta distinción con la habitual entre explanans y explanandum en una explicación particular. La distinción propuesta es relativa al papel de los conceptos en una teoría, y no relativa a explicaciones particulares, y estos diferentes papeles pueden no coincidir (y de hecho no coinciden). Esto es importante, porque la tesis de este trabajo es que se ha asumido incorrectamente que la base de contrastación global coincide con el explanandum global de la teoría, y que el explanans global coincide con el complemento de la base de contrastación global (que no tiene un nombre específico en la literatura). Esto es compatible con que en contrastaciones y explicaciones particulares en el explanans local aparezcan términos que no pertenecen al explanans global (p.ej. en las condiciones iniciales, en el enfoque clásico pueden aparecer condiciones iniciales observacionales) o que en una contrastación particular aparezcan como dados, en lo que Gähde ha llamado "aplicaciones secundarias" de una teoría (1996), conceptos teóricos a través de vínculos entre modelos de una misma teoría (razón por la cual, justamente, Lorenzano propone la noción de "base empírica global"). El uso de "explanans" en el sentido de explanans global de una teoría no es habitual en la literatura, y justamente el objetivo de este trabajo es introducir dicha noción. Para la comprensión de este trabajo es importante no confundir la discusión respecto al papel de los conceptos en explicaciones o contrastaciones locales, y el papel de los conceptos en una teoría, en sentido global. Este trabajo trata de esta última cuestión. También es importante resaltar que al hablar de "explanans global" para referirnos al conjunto de conceptos explicativos en una teoría particular, no queremos implicar que estos por sí mismos sean un componente explicativo suficiente. Dado que, en las leyes, en el marco estructuralista, se encuentran relacionados todos o casi todos los 
Díez es todavía más explícito en este punto cuando sostiene:

Llamaremos vocabulario T-contrastacional, o T-no-teórico, o T-empírico, a aquella parte del vocabulario característico de la teoría $T$ usado en la descripción de los " $T$-datos", esto es, de los "fenómenos" de los que la teoría quiere dar cuenta (explicar/predecir) [...] Llamaremos vocabulario T-explicativo, o $T$-teórico, al vocabulario característico de $T$ que no es vocabulario $T$-contrastacional, esto es, a los conceptos usados en la formulación de las leyes de $T$ que no se pueden determinar/medir sin presuponer la validez de alguna de dichas leyes. (2012a, pp. 68-69)

La identificación de la base empírica global de contrastación y el explanandum global de la teoría se presupone en el propio marco conceptual estructuralista, en la caracterización del concepto de aplicación pretendida de una teoría - aquellos sistemas de los que la teoría quiere dar cuenta, es decir, cuyo comportamiento se pretende explicar con la teoría - . Pues se las caracteriza como modelos potenciales parciales, que surgen, técnicamente, de recortar los conceptos T-teóricos a los modelos potenciales, en donde aparecen todos los conceptos de la teoría (Balzer et al. 1987, p. 86; Díez y Lorenzano 2002, pp. 64-65). En resumen, todos los conceptos T-no teóricos de una teoría formarían parte del explanandum global de la teoría. Aquí hay dos supuestos: el primero es que el explanandum de la teoría se expresa en términos no teóricos, lo cual es razonable, pues se espera que el explanandum de la teoría se exprese independientemente de la teoría y que la preceda, desde un punto de vista lógico y también, usualmente, desde un punto de vista histórico. El otro supuesto, que es el que discutiremos, es que en los casos en los que hay ampliación conceptual, es decir, en casos en los que las aplicaciones pretendidas son enriquecidas conceptualmente, tal enriquecimiento apele sólo a términos T-teóricos (además de términos lógico-matemáticos, por supuesto).

La identificación entre las distinciones según la explicación y la contrastación se presupone también en algunos escritos donde, tratando de elucidar la noción de explicación genuina, se apela explícitamente a la T-teoricidad de los conceptos. Así, por ejemplo, Moulines afirma:

conceptos de la teoría, la explicación por subsunción nómica implica la interrelación de todos los conceptos, teóricos y no teóricos, o explicativos y no explicativos, para que la magia se produzca. 
Si aceptamos que tiene sentido hacer una distinción entre conceptos T-teóricos y conceptos T-no-teóricos dentro de una teoría T, y si admitimos una interpretación realista de los conceptos T-teóricos, entonces [... ] las entidades teóricas a las que se refieren los conceptos T-teóricos se introducirían [...] para proporcionar una explicación causal de los procesos fenoménicos codificados en nuestros modelos de datos. Ellas serían parte de la estructura causal "oculta" del mundo que es responsable de los fenómenos que observamos y codificamos por medios T-no-teóricos. (2005)

De este texto nos interesa resaltar, no tanto la cuestión de la causalidad, que también es discutible (Lorenzano 2005), sino la clara referencia a que los conceptos T-teóricos serían explicativos en las teorías en las que aparecen. Por su parte, Díez sostiene que:

la explicación de los fenómenos, T-no teóricamente identificados, la proporcionan los constituyentes T-teóricos de los modelos. Es porque en el sistema están "presentes" esos constituyentes T-teóricos comportándose con los T-no teóricos como las constricciones establecen, por lo que entendemos el comportamiento de la parte T-no teórica del sistema. (2002).

En el trabajo citado, Díez identifica las teorías que enriquecen conceptualmente sus aplicaciones pretendidas con fines explicativos con aquellas que proponen conceptos teóricos. Y las teorías que no enriquecen conceptualmente sus aplicaciones pretendidas con las que carecen de términos teóricos. Bajo esta perspectiva, el enriquecimiento conceptual de las aplicaciones pretendidas siempre se da a través de términos teóricos. En un artículo posterior Díez (2014) ha debilitado esta posición en un sentido relevante que discutiremos posteriormente.

\section{Hacia una distinción entre contrastabilidad y explicatividad}

La tesis principal de este artículo es que la explicatividad y la contrastabilidad, que han sido tratadas como uno y el mismo problema en los sentidos señalados anteriormente, deben ser diferenciadas. Con este fin introduzcamos una distinción análoga aunque distinta a la Tteoricidad, que como hemos venido sosteniendo, tiene que ver con la contrastabilidad de una teoría: la T-explicatividad. Por supuesto, estrictamente hablando, los conceptos por sí mismos no explican nada. La idea es hablar del papel explicativo de un concepto en una teoría 
en sentido global (no en aplicaciones particulares). Los conceptos Texplicativos (explicativos en T) serían aquellos conceptos con los que la teoría amplía conceptualmente sus aplicaciones pretendidas (aquellos sistemas cuyos comportamientos la teoría pretende explicar). Los conceptos con los que se describen los sistemas de cuyo comportamiento se pretende dar cuenta en T serían los T-no explicativos (no explicativos en T). En una teoría, los conceptos explicativos suelen ser los que describen factores causales, pero no siempre, porque existen teorías explicativas, que amplían conceptualmente sus aplicaciones pretendidas, pero que no proveen explicaciones causales (Lorenzano 2005).

Como veíamos, se supone habitualmente que la distinción de Tteoricidad permite distinguir entre los conceptos cuya determinación independiente posibilita la contrastación independiente de la teoría, de aquellos que no, y además, entre aquellos conceptos que tienen un papel explicativo de los que no. Es decir, bajo el punto de vista en discusión, la T-teoricidad es intensional y extensionalmente coincidente con la T-explicatividad. Nosotros intentaremos defender que ambas distinciones no coinciden ni intensional ni extensionalmente.

Insistimos, la crítica de los autores mencionados en la sección 2 permitió notar que la observabilidad no desempeña un papel esencial en la determinación de la base "empírica" (en el sentido de base de contrastación global) de una teoría y esa mejora fue introducida en el criterio estructuralista de T-teoricidad. Es posible señalar una confusión semejante en lo que respecta a la forma en que se utiliza la distinción de T-teoricidad, pues, como hemos señalado en el punto anterior, se suelen confundir dos distinciones, la que se refiere a la contrastabilidad y la que se refiere a la explicatividad. El criterio propuesto por los estructuralistas para la T-teoricidad permite distinguir, en consecuencia, la base de contrastación de una teoría, pero no el conjunto de posibles aplicaciones pretendidas de la teoría, y en consecuencia, tampoco el conjunto de conceptos que constituyen las aplicaciones pretendidas actuales, es decir, el explanandum global de la teoría.

El punto es que no se ha dado ninguna razón para considerar que el hecho de que un concepto no pueda ser determinado independientemente de una teoría pueda servir para determinar el papel explicativo de tal concepto en esa teoría. Y en caso de que ambas distinciones fuesen coextensivas, no se han dado razones para sostener que esta coincidencia no sea contingente. Pues es posible imaginar teorías que expliquen apelando a conceptos disponibles con anterioridad, y, por lo tanto, no teóricos en la teoría en cuestión. Pero, 
cambiemos la carga de la prueba ¿existe alguna razón para sostener que, efectivamente, las distinciones — según la teoricidad y según la explicatividad - no coinciden intensionalmente ni extensionalmente?

Nótese primero lo siguiente: como ya hemos señalado, un concepto es teórico para una teoría $\mathrm{T}$ dada si todos los métodos de determinación del concepto presuponen T. Ahora bien, ¿existe alguna razón por la cual sería imposible el descubrimiento de un nuevo método de determinación independiente de $\mathrm{T}$ de un concepto que hasta el momento era T-teórico? Si no es imposible, entonces, estamos obligados a aceptar que un concepto T-teórico puede volverse T-no teórico sin ningún cambio en la teoría de la cual era T-teórico. La distinción, en consecuencia, puede variar con el tiempo. Esta posibilidad está contemplada, por ejemplo, en An Arquitectonic for Science para el concepto de masa. Allí se afirma que

La masa de un cuerpo se daría [en una teoría independiente acerca de la estructura de la materia] sólo por el número de sus átomos [...]. No hay objeciones formales contra este procedimiento, pero es difícil ver cómo esta convención podría aplicarse empíricamente. No podemos contar los átomos de un cuerpo macroscópico. Una dificultad adicional es que, de acuerdo con la química daltoniana, los átomos de diferentes sustancias tienen diferentes masas. Uno puede ciertamente imaginar que otra convención más refinada del tipo de "cantidad de materia" finalmente podría conducirnos a la definición correcta de masa que sea además aplicable prácticamente. Pero, por ahora, no sabemos de ninguna propuesta por el estilo. (Balzer et al. 1987, p. 53; la traducción es nuestra.)

Como sugiere la cita, sólo podemos afirmar que por ahora el concepto de masa es T-teórico. Si éste es el caso, la cuestión resulta relevante, pues los conceptos en una teoría dada podrían cambiar su estatus de teoricidad por el descubrimiento de nuevos métodos de determinación, y esto no tendría por qué cambiar su estatus respecto a la explicatividad. En las aplicaciones pretendidas de la mecánica clásica encontramos partículas con diferentes aceleraciones. Para dar cuenta de los movimientos de las partículas en la mecánica clásica se apela a fuerzas y masas. Fuerza y masa serían, en consecuencia, y según la forma en que introdujimos la distinción, Mecánica Clásicaexplicativos $^{5}$ ¿Dejaría la masa de funcionar como explicativa en este

${ }^{5}$ Se ha señalado que masa no cumple un papel explicativo (o causal) en la mecánica clásica (Barberis 2013, pp. 78-79; Forge 2002, p. 113). La idea es que el papel de la masa de la partícula es la de especificación del estado antecedente, pero 
sentido en caso de que se descubriera un método independiente de la Mecánica Clásica para su determinación? No parece haber razones (salvo la identificación a priori de la explicatividad y la contrastabilidad) para hacerlo. Fuerza y masa seguirían siendo T-explicativas, pero la masa se volvería no teórica.

Esto permite sospechar que la distinción entre la T-teoricidad y la T-explicatividad no son intensionalmente coincidentes. Por supuesto, aunque no sean intensionalmente coincidentes, las distinciones podrían coincidir en todos los casos por alguna razón no conceptual. Hasta el momento, sin embargo, nadie ha dado ninguna razón de este tipo, simplemente porque las distinciones no han sido diferenciadas con claridad. Tomar conciencia de que estamos frente a distinciones diferentes, en todo caso, abre la posibilidad de que se inicie un debate al respecto.

Pero, además, consideramos que tampoco hay razones para que las distinciones deban coincidir intensionalmente, porque, según nuestro punto de vista, no coinciden extensionalmente. Es posible encontrar teorías, en la historia de la ciencia, que han enriquecido conceptualmente sus aplicaciones pretendidas con conceptos que pueden ser determinados de manera independiente. Es decir, existen casos de teorías que explican apelando a conceptos no teóricos. Repasaremos algunos en la sección siguiente.

\section{Explicando con conceptos no teóricos}

Daremos algunos ejemplos de teorías que se han reconstruido recientemente y en las que la distinción de T-teoricidad y de Texplicatividad no coincide, o bien, no es claro que coincidan: la teoría de la selección natural de Darwin (y la de Wallace), la teoría del origen común de Darwin, la genética de poblaciones, la teoría de la anomia de Merton y la teoría del enlace valencia. Por supuesto, por cuestiones de espacio, las presentaciones serán esbozadas, informales

la carga explicativa de la aceleración se encontraría en la fuerza. Si bien es posible contraargumentar que la masa de la partícula es explicativa de que la partícula adquiera cierta aceleración, en realidad el punto no es relevante en este caso. A las aplicaciones pretendidas de una teoría se les ha caracterizado como los fenómenos de los que las teorías quieren dar cuenta. En la descripción de tales fenómenos, tal como se presenta la cuestión habitualmente, no figuran las masas respectivas. Hemos dicho que los conceptos T-explicativos son aquellos conceptos descriptivos con los que se enriquecen conceptualmente las aplicaciones pretendidas en una teoría dada T. Esto basta para señalar que masa es T-explicativo, y que ese papel no cambiaría si se encontraran métodos de determinación independientes de la masa, volviéndose ésta no teórica. 
y resumidas. Para que esta sección sea convincente, los análisis presupuestos deben serlo, y para eso, no queda sino introducirse en las reconstrucciones particulares en cuestión. En este trabajo asumiremos la adecuación de las reconstrucciones. Si hubiera dudas respecto a las reconstrucciones el lector debería apelar a los textos en los que las reconstrucciones se presentaron de manera completa.

\section{a. Teoría de la selección natural}

La naturaleza y estructura de la teoría de la selección natural ha sido un tema de discusión importante en la filosofía de la biología. Para el punto que queremos discutir nos basaremos en la reconstrucción que Ginnobili hizo en términos estructuralistas (2010, 201la, 2012). Aquí no incluiremos los detalles puntuales y discutibles de la reconstrucción que se ofreció. Baste señalar que la ley fundamental de la teoría sería, según Ginnobili, la siguiente: "Los organismos que portan un rasgo $r$ que cumple más efectivamente una función en un ambiente determinado $a$ tienden a mejorar su aptitud en $a$, tendiendo a mejorar en consecuencia, si $r$ es heredable, el éxito reproductivo diferencial en $a$ de este tipo de organismos."

La aptitud sería un concepto abstracto que se especificaría de diferentes modos en las diferentes leyes especiales que conforman la teoría. Los siguientes serían diferentes casos de aplicación, algunos de Darwin y otros posteriores, que surgen de las diferentes especificaciones del concepto de aptitud como supervivencia, como capacidad de apareamiento, como fecundidad, etc.:

- Las jirafas con cuello, patas delanteras, cabeza y lengua de mayor longitud son más efectivas al alimentarse de las ramas más altas de los árboles, mejorando su supervivencia y mejorando, en consecuencia, su éxito reproductivo diferencial (Darwin 1872, p. 178).

- Los gallos de espolones más efectivos para luchar con otros gallos tienden a lograr emparejarse más, mejorando, en consecuencia, si el rasgo es heredable, su éxito en la reproducción diferencial (Darwin 1859, p. 88).

- Las plantas que producen flores más atractivas a los insectos tienden a mejorar su fecundidad mejorando, en consecuencia, si el rasgo es heredable, su éxito en la reproducción diferencial (Darwin 1859, p. 92). 
- Las polillas de alas de color más oscuro se mimetizan mejor con su ambiente a los ojos de sus predadores, mejorando su supervivencia, mejorando, si el rasgo es heredable, su éxito en la reproducción diferencial (Kettlewell 1955, 1956).

Lo que esta teoría explica es por qué cierto tipo de organismos en una población tiene un mayor éxito reproductivo que otro. Las aplicaciones pretendidas de la teoría, en consecuencia, son organismos en una población que difieren en la posesión de un rasgo y que difieren en su éxito reproductivo. Los conceptos con los que se enriquecen conceptualmente las aplicaciones pretendidas, y en consecuencia Texplicativos, son: función, aptitud, heredabilidad, efectividad al cumplir una función. ${ }^{6}$

Ahora, como señala el autor, es bastante sencillo mostrar que al menos algunos de los conceptos que cumplen un papel explicativo en la teoría, son no teóricos en esa teoría: que un rasgo de un organismo cumple una función es determinable desde la biología funcional, la efectividad con la que ese rasgo cumple su función también es determinable independientemente de la teoría, y, por supuesto, la determinación de que un rasgo es heredable tampoco la presupone. El único concepto que podría funcionar como teórico en la teoría sería el de aptitud. Esto es esperable, además, porque de la especificación de tal concepto es que surgen las diferentes

${ }^{6}$ Podría objetarse que lo que se quiere explicar varía según el contexto explicativo, de acuerdo con la pregunta "por qué" que se realice. Por ejemplo, si preguntáramos "¿Por qué los organismos que poseen un rasgo que cumplen cierta función efectivamente tienen un mayor éxito reproductivo?" parecería que el concepto de función formaría parte del explanandum de la teoría, y no del explanans como se sostiene en el análisis presentado. Esto sería grave para nuestra propuesta de distinguir entre conceptos T-explicativos y T-no explicativos, puesto que la distinción no podría trazarse con claridad. Pueden hacerse dos comentarios al respecto. Por un lado, de este modo no se salvaría la coincidencia extensional e intensional de las distinciones de T-teoricidad y de T-explicatividad. Pues, la T-explicatividad variaría según el contexto mientras que la T-teoricidad no. Sin embargo, creemos que éste es un modo inadecuado de defender nuestro punto. Pues se estarían confundiendo la distinción local de qué explica qué en un contexto particular, con la cuestión global de qué explica una teoría. Parece adecuado sostener que lo que la teoría de la selección natural explica (en términos globales) son las diferencias en el éxito reproductivo entre diferentes tipos de organismos. O al menos, en las reconstrucciones estructuralistas, se considera que tiene sentido, planteada de manera global e independientemente de contextos particulares, el intento de establecer cuáles son las aplicaciones pretendidas de una teoría. Y esto, como hemos tratado de defender, tiene que ver con que es aquello que se quiere explicar con la teoría. La misma objeción y la misma contraobjeción se podrían realizar respecto a los otros casos analizados. 
leyes especiales, algo que suele ocurrir con algunos términos teóricos (como fuerza en la Mecánica Clásica). Sin embargo, el punto del autor es más fuerte, pues sostiene que incluso el concepto de aptitud puede ser catalogado como no teórico. Pues sus especificaciones (la supervivencia, la fecundidad, etc.) pueden también determinarse con independencia de la teoría. Este último punto es discutible, pues podría sostenerse que es gracias a la teoría de la selección natural que todas estas especificaciones caen bajo el mismo concepto de aptitud, y en definitiva, esto podría implicar que no se puede determinar el concepto de aptitud sin la teoría de Darwin. Aunque este fuese el caso, seguiría ocurriendo que entre los conceptos T-explicativos figuran algunos términos T-no teóricos.

Por otra parte, como lo ha señalado Ginnobili en otra oportunidad, aunque aptitud fuese teórico, la historia de la ciencia nos brinda otro ejemplo a tener en cuenta: la versión original de Wallace de la teoría de la selección natural (Ginnobili 2011b, pp. 219-222). Wallace propone una teoría semejante a la de Darwin, que tiene una única diferencia: carece de un concepto general de aptitud. Todas las aplicaciones de Wallace implican una mejora en la supervivencia (Ginnobili y Blanco 2010). La ley fundamental de la teoría de Wallace, sería, en consecuencia, idéntica a la ley especial de la teoría darwiniana en la que se especifica el concepto de aptitud como supervivencia. En este último caso, la ampliación conceptual de las aplicaciones pretendidas no sería mixta (en el sentido de incluir términos teóricos y no teóricos) como podría ocurrir en la teoría de Darwin si aptitud es considerado como teórico, sino no teórica (en el sentido de que apelaría únicamente a términos descriptivos no teóricos).

En cualquier caso, hay buenas razones para pensar que en esta teoría la distinción según la contrastabilidad y la distinción según la explicatividad, no coinciden.

\section{b. Teoría del origen común}

Seguiremos en este caso la reconstrucción que hace Blanco (2012). La teoría del origen común, que parece haber sido la teoría más cara al propio Darwin, permite explicar un fenómeno que había sido conceptualizado previamente a los trabajos de Darwin: las semejanzas homológicas entre diferentes organismos. La teoría afirma que las semejanzas homológicas (como el parecido estructural que existe entre nuestra mano y la aleta de una ballena o el ala de un murciélago) se explican apelando a que tales rasgos homólogos fueron heredados de un mismo antepasado. 
La ley fundamental de la teoría del origen común, algo simplificada, según Blanco:

Para cualquier par de organismos, si dos rasgos son semejantes entre sí estando presentes un rasgo en uno de los organismos y el otro en el otro, entonces, existe un tercer organismo que posee un rasgo tal que (1) este rasgo es semejante a los dos primeros; (2) este tercer organismo es ancestro de ambos; (3) el rasgo es heredable.

Nótese que en este caso el campo de aplicaciones pretendidas está constituido por organismos de diferente tipo que tienen rasgos que exhiben semejanzas homológicas. Para dar cuenta de esta semejanza Darwin apela a los conceptos T-explicativos de heredabilidad y ancestría. Como el mismo Blanco señala, ninguno de estos conceptos parece teórico en la teoría del origen común, éste sería otro caso de ampliación conceptual no teórica. Pues que cierto organismo es ancestro de otro, como que cierto rasgo es heredable, puede ser determinado en prescindencia de la teoría del origen común.

\section{c. Genética de poblaciones}

Existen numerosas reconstrucciones de la genética de poblaciones que han seguido caminos heterogéneos. Nos centraremos en la que Lorenzano realizó, presuponiendo las herramientas del estructuralismo metateórico (2014). En este trabajo Lorenzano muestra convincentemente que el principio de Hardy-Weinberg no puede ser la ley fundamental de la genética de poblaciones. Esta reconstrucción tiene de original, frente a otras, que se presenta a la genética de poblaciones como una extensión de la genética clásica.

Como el mismo Lorenzano afirma, las aplicaciones pretendidas de la genética de poblaciones serían los cambios en las distribuciones de características en una población dada, en diferentes generaciones (2014, p. 164).

Para dar cuenta de tales cambios se apelaría a la siguiente ley fundamental (presentada de manera informal y de manera simplificada):

La distribución estadística de rasgos entre los miembros de una población es debida a la presencia de genotipos en los miembros que "producen" (en conjunto con el ambiente) tales rasgos y a los factores evolutivos que pueden afectar la distribución probabilística de los genotipos de los miembros de la población entre generaciones, de modo que la distribución de genotipos coincide de una manera específica con la distribución de rasgos. 
Si bien la teoricidad de los conceptos no se trata de manera explícita en este trabajo, resulta bastante claro que, en esta versión en la que se presenta a la genética de poblaciones como una extensión de la genética clásica, habría algunos conceptos teóricos. Aquellos que describen los genotipos, la obtención de unos genotipos a partir de otros, en la descendencia, y la relación entre genotipo de fenotipo. Ahora bien, las aplicaciones pretendidas (que incluiría la descripción de los cambios en las frecuencias de rasgos en una población), no serían ampliadas conceptualmente sólo con estos conceptos teóricos, sino que también se apela a los factores evolutivos, como la migración, la deriva génica, la selección natural y la mutación. Dejando de lado la selección natural que podría ofrecer algún tipo de discusión (pues algunos asumen que la selección natural es una parte de la genética de poblaciones), y la deriva génica, cuyo estatus es también discutible y de hecho discutido, es bastante claro que la migración y la mutación son no teóricos en la genética de poblaciones. Pues, es posible determinar tales conceptos en prescindencia de ella. En este sentido, estamos frente a otro caso de ampliación conceptual mixta.

Si se reconstruyera la genética de poblaciones como diferente (aunque vinculada) con la genética clásica, de modo que la ley fundamental afirmara (esbozadamente) algo como lo que sigue:

La distribución de genotipos a lo largo de diferentes generaciones sufre modificaciones por la acción de la deriva génica, la selección natural, la mutación y la migración.

En ese caso nuestro punto se fortalece, ya que no quedaría claro que entre los conceptos con los que se amplía conceptualmente las aplicaciones pretendidas exista algún término teórico para la genética de poblaciones. Lo mismo puede sostenerse, incluso, si se ofreciera una reconstrucción en la que el principio de Hardy-Weinberg fuese la ley fundamental. Excede el espacio disponible discutir con más precisión tales posibilidades. La cuestión central es que la afirmación de que la genética de poblaciones no se amplía conceptualmente apelando sólo a conceptos teóricos se mantendría en todas estas diferentes estrategias reconstructivas.

\section{d. Teoría de la anomia}

Nos basaremos para la presentación de la teoría en la presentación y reconstrucción de Abreu (2014). Merton intenta explicar lo que llama "conductas desviadas" de individuos en una sociedad a partir de una explicación social, en contraposición con la idea de que tales 
conductas se deben en realidad a cuestiones biológicas o psicológicas individuales (1938). La explicación de tales conductas involucra la distinción entre los objetivos culturalmente asumidos como importantes y los medios disponibles para lograr tales objetivos. Los individuos asimilan dichos objetivos y las normas que dicta la moral de la sociedad en cuestión. Frente a la imposibilidad de cumplir dichos objetivos, los individuos deben adaptarse. Hay cuatro tipos de adaptación posibles:

a) Innovación, que corresponde a seguir los objetivos culturalmente aceptados abandonando los medios aceptables para lograr tales objetivos.

b) Ritualismo, cuando se abandonan los objetivos socialmente valorados, cayendo en la aceptación de los medios como un fin en sí mismo.

c) Retraimiento, que corresponde al abandono de los objetivos socialmente valorados, y de los medios aceptables para lograrlos.

d) Rebelión, que implica el abandono de los objetivos socialmente valorados y de los medios aceptados, pero además, presupone el intento de legitimar una nueva conformación social, en la que se revisan objetivos y normal.

Estas adaptaciones serían casos de inconformidad. El individuo que acepta los objetivos culturalmente aceptados y los medios socialmente aceptables para lograrlos, presentarían una conducta conformista, que es la que habitualmente prevalece.

La ley fundamental de la teoría de la anomia, simplificada, sería:

Cuando existe un conflicto entre objetivos y medios, y el individuo no logra un objetivo socialmente valorado, se espera que presente una conducta inconformista, debido a (y según sea) la asimilación que haga de la importancia del objetivo y de las normas que regulan los modos aceptables de lograrlo.

Lo que la teoría pretende explicar, tal como Abreu afirma, son conductas de individuos particulares, como individuos que roban, que se copian en un examen, que no participan en absoluto de la sociedad, o que acumulan dinero como un fin en sí mismo. Abreu no distingue, como sí ocurre en las reconstrucciones de los dos casos anteriores, entre las aplicaciones pretendidas y los modelos potenciales parciales 
(los modelos que se obtienen de recortar los conceptos teóricos de los modelos que conforman la teoría). En consecuencia, cuando presenta las aplicaciones pretendidas incluye todo lo que considera no teórico en la teoría de la anomia. Pero es fácil ver que la descripción previa a la ampliación conceptual de la teoría de la anomia sólo tiene individuos que tienen ciertas conductas. A esa descripción preteórica se la amplía con las nociones de objetivos culturalmente aceptados, medios aceptables, asimilación de objetivos y de medios, y adaptaciones. Todos estos conceptos serían explicativos en la teoría de la anomia, es decir, T-explicativos. Ahora bien, según Abreu, el único concepto que tendría sentido caracterizar como teórico en tal teoría, sería el de asimilación de normas y objetivos. Pues, según afirma, no habría manera de determinar la asimilación de un individuo sino a partir de la forma en que tal asimilación es conectada con la conducta del individuo en la ley fundamental de la teoría de la anomia. Tal vez la teoricidad de la función de asimilación sea discutible, pero no es necesario hacerlo. Si las cosas son como las presenta Abreu, estaríamos frente a un caso de ampliación conceptual mixta, en donde se amplía conceptualmente apelando a conceptos teóricos y no teóricos. éste sería otro caso en el que la T-teoricidad y la T-explicatividad no coincidirían.

\section{e. Teoría del enlace de valencia}

Nuestro último ejemplo lo constituirá una teoría de la química. La teoría del enlace de valencia la presentaron y desarrollaron varios autores (Heitler y London 1927; Pauling 1931; Slater 1929) con la meta de aproximar la mecánica cuántica a la química. La teoría fue reconstruida recientemente en un trabajo que muestra la relevancia de las reconstrucciones en la comunicación y la enseñanza de la ciencia (Ariza 2015). La teoría explica la naturaleza de los enlaces a través del solapamiento de orbitales atómicos ocupados por un solo electrón, y, consecuentemente, a la combinación de los espines electrónicos de cada electrón, determinando su valencia.

La ley fundamental de la teoría afirma:

Si los orbitales híbridos o puros que se solapan tienen un solo electrón respectivamente y el espín del electrón de cada átomo es opuesto, se cumple que la valencia de los átomos será el número de orbitales solapados con un electrón del átomo que participa en el enlace (Ariza 2015, p. 91). 
Las aplicaciones pretendidas de la teoría, en consecuencia, son descriptas a través de los siguientes conceptos: átomo, enlace (de átomos formados por núcleos y electrones) para compartir electrones. Para dar cuenta de la naturaleza del enlace valencia, es decir, de por qué dos átomos se unen para compartir electrones, la teoría apela a una serie de conceptos: orbital atómico (híbrido o puro), solapamiento, espín (entre otros). Entre estos conceptos, el autor reconoce algunos conceptos teóricos para la teoría. En particular, el concepto de solapamiento que constituye la contraparte teórica de la noción previa de enlace y la noción de valencia. Ambas nociones requieren la teoría para ser aplicadas. Pero, las aplicaciones pretendidas no son enriquecidas únicamente con conceptos teóricos, pues también se apela a conceptos que claramente provienen de y pueden ser determinados desde otras teorías. Por ejemplo, el concepto de espín, que proviene de la mecánica cuántica. Lo mismo ocurre según Ariza con el concepto de orbital atómico, respecto del cuál afirma explícitamente que no forma parte de los modelos parciales de la teoría aunque es utilizado para dar cuenta de sus aplicaciones pretendidas (2015, p. 93), no al menos, según el criterio de T-teoricidad que nos interesa. En conclusión, sería ésta una teoría que enriquece conceptualmente sus aplicaciones pretendidas apelando tanto a conceptos teóricos como no teóricos en la teoría. Sería otro caso de ampliación conceptual mixta.

\section{Consecuencias para el estructuralismo}

Existen consecuencias interesantes que pueden extraerse de lo anterior relacionadas con el estructuralismo metateórico. Es importante tener en cuenta que las modificaciones en el aparato conceptual del estructuralismo son usuales. Pues, el estructuralismo es un programa metateórico en un periodo "normal" que habitualmente involucra la aplicación a nuevos casos, y a veces, modificaciones no esenciales en su aparato conceptual. Lo que hemos señalado no constituye, en este sentido, una anomalía. Finalmente, nos gustaría señalar que las modificaciones sugeridas no tienen que ver con cuestiones esotéricas o internas al estructuralismo, sin ninguna relevancia para aquel que no pertenece al programa. No, nuestra idea es que la sofisticación y precisión del marco conceptual estructuralista es la que nos ha permitido señalar, si tenemos razón, una cuestión relevante para toda la filosofía de la ciencia, señalando cuestiones interesantes y generales respecto de la contrastación y la explicación.

Presentaremos dos consecuencias para el estructuralismo: 


\section{a. Aplicaciones pretendidas como modelos potenciales parciales:}

El concepto de aplicaciones pretendidas es tomado por el estructuralismo metateórico de la modificación que Adams (1955) hizo al procedimiento de Suppes de axiomatización de teorías apelando a la introducción de un predicado conjuntista en términos de la teoría de conjuntos (1970). La idea consiste en que la presentación del núcleo formal de una teoría no alcanza para identificarla. Es necesario indicar a qué fenómenos pretende aplicarse dicho núcleo. La determinación del conjunto de aplicaciones pretendidas tiene un elemento pragmático ineliminable, pues es esencialmente intencional, dado que depende de la intención de la comunidad científica de que el núcleo se aplique a ciertos fenómenos, y es también paradigmática, porque no se define extensionalmente presentando una lista de todos sus elementos, sino a través de relaciones de semejanza con un subconjunto de ejemplares paradigmáticos (herencia clara de Kuhn).

En las presentaciones habituales del estructuralismo no sólo se requiere que las aplicaciones pretendidas de $\mathrm{T}$ se describan con lenguaje no teórico de $\mathrm{T}$, lo cual es un requerimiento razonable, puesto que deben poder caracterizarse con independencia de la teoría, sino que se sostiene que las aplicaciones pretendidas (I) son un subconjunto de los modelos potenciales parciales (Mpp) (Díez y Lorenzano 2002, pp. 64-65). Los Mpp, por su parte, se obtienen técnicamente a través de una función que recorta, justamente, los elementos Tteóricos de T (Díez y Lorenzano 2002, pp. 62-64). Ahora bien, si las aplicaciones pretendidas de una teoría son aquellos fenómenos de los cuales se quiere dar cuenta con la teoría (Díez y Lorenzano 2002, p. 64), el hecho de que existan casos de ampliación conceptual mixta (que las aplicaciones pretendidas se amplíen conceptualmente con términos teóricos y no teóricos en esa teoría) o puramente no teórica (que haya ampliación conceptual pero sin apelar a conceptos teóricos para la teoría) conduce a dificultades con la forma de caracterizar las aplicaciones pretendidas. Pues, las aplicaciones pretendidas en estos casos serían en realidad estructuras parciales de los Mpp (es decir, surgen de recortarle a los Mpp ciertos conceptos). ${ }^{7}$ Esto no es una modificación muy importante dentro del aparato del estructuralismo

\footnotetext{
${ }^{7}$ Otra opción podría consistir en realizar la distinción entre modelos potenciales y modelos potenciales parciales según la distinción propuesta de T-explicatividad, de modo que las aplicaciones pretendidas sigan siendo un subconjunto de los Mpp. Si bien ésta es una cuestión estrictamente terminológica, preferimos la opción más conservadora de seguir trazando la distinción entre Mp y Mpp con base en la Tteoricidad porque, por una parte, implica menos cambios en el modo de hablar de los estructuralistas metateóricos, y por otra, porque consideramos que la distinción
} 
metateórico, pues sólo implica caracterizar al conjunto I de un modo ligeramente distinto, como una estructura parcial de los Mpp. Esta distinción, seguramente, podrá ser otra de las cuestiones detrás de las cuales se agazapará la pragmática (Díez y Lorenzano 2002, pp. 99115).

\section{b. Explicación como subsunción}

Como mencionamos anteriormente, Díez (2012b, 2014) elaborando el trabajo comenzado por otros autores estructuralistas (Bartelborth 1996, 2002; Forge 2002), intenta brindar una nueva elucidación del concepto de explicación científica que supere tanto la versión unificacionista (Kitcher 1981) como la causalista (Salmon 1998). A nuestro juicio, este nuevo enfoque respecto de la explicación es tan fructífero como Díez sugiere, y además, supera los enfoques alternativos teniendo en cuenta los objetivos que estos mismos se plantean. No defenderemos, sin embargo, esta cuestión en este trabajo. Nos limitaremos a mostrar la relevancia de las distinciones realizadas aquí para el enfoque de Díez.

En el enfoque que Díez propone, de subsunción teórica ampliativa, explicar consiste en subsumir el fenómeno en un patrón nómico, esto es, en los términos modelo-teóricos, en subsumir el fenómeno en una rama de la red teórica de constricciones nómicas de la teoría. La explicación debería cumplir dos condiciones necesarias según Díez, por una parte, que la explicación se realice a través de las leyes especiales pertinentes, y por otra, que la explicación amplíe conceptualmente las aplicaciones pretendidas de las que se pretende dar cuenta. Nuestro interés radica en este último requisito. Lo que se pretende elucidar con éste es la intuición razonable de que las teorías fenomenológicas (las que no enriquecen conceptualmente, sino que meramente generalizan) no son tan explicativas como las no fenomenológicas. En los textos en los que Díez introduce esta idea (2012b), siempre considera que las teorías que amplían conceptualmente sus aplicaciones pretendidas lo hacen sólo a través de conceptos T-teóricos (considerando, por supuesto, únicamente los conceptos descriptivos).

Al examinar los ejemplos que Díez presenta, las teorías que tiene en mente cuando habla de teorías que tienen algún déficit explicativo

original con base en la T-teoricidad, y el recorte con base en tal distinción sobre los modelos potenciales, para obtener los modelos potenciales parciales, tiene que ver específicamente con la cuestión más clásica de cómo el significado de un concepto teórico está dado en parte por sus métodos de determinación, cuestión para la cuál es relevante la T-teoricidad y no la T-explicatividad. 
respecto al criterio que nos interesa, son las que no extienden conceptualmente sus aplicaciones pretendidas, como es el caso de la ley de caída libre de Galileo o las leyes de Kepler. Pero hemos visto casos de teorías que amplían conceptualmente con conceptos teóricos y no teóricos en la teoría, y casos de teorías que amplían conceptualmente sin apelar a conceptos teóricos para la teoría. Tales casos, si lo que se pide como requisito es que la ampliación sea únicamente en términos teóricos, presentaría también algún déficit explicativo. Esto nos parece inadecuado porque las teorías que hemos visto, que amplían no teóricamente, o de manera mixta (apelando a conceptos teóricos y no teóricos) parecen brindar explicaciones genuinas. Por otra parte, no es que Díez vea un problema en estas teorías, sino que sencillamente no tuvo en cuenta la posibilidad de que las teorías amplíen conceptualmente con conceptos no teóricos, sencillamente porque esa posibilidad no se ha tenido en cuenta ni dentro del estructuralismo, ni en general.

En un artículo más reciente, por sugerencia nuestra, Díez ha considerado la posibilidad de que exista ampliación mixta (2014). Según afirma, en teorías con relaciones interteóricas complejas, como ocurriría justamente con la teoría de la selección natural, podría ocurrir que la ampliación conceptual incluyera conceptos no teóricos, además de los teóricos. En este sentido ha aceptado como requisito para que una explicación sea genuina, la ampliación conceptual mixta. La concesión de este punto ya alcanza a mostrar lo que pretendemos mostrar, que es que la distinción de la T-explicatividad y la distinción de T-teoricidad no son ni intensionalmente ni extensionalmente coincidentes. En consecuencia, las modificaciones que el estructuralismo debiera hacer en su marco, señaladas en el punto anterior, ya serían necesarias.

Sin embargo la siguiente cuestión permanece. ¿Por qué para que una explicación sea genuina debería ampliarse conceptualmente con al menos algún concepto teórico en esa teoría? La razón por la cuál es razonable exigir ampliación conceptual como bien señala Díez, tiene que ver con la intuición compartida con el análisis causalista de que explicar implica apelar a algo ontológicamente nuevo (2012b, p. 553). Pero, ¿por qué la novedad ontológica debiera categorizarse con conceptos teóricos en la teoría en cuestión?

En comunicación personal Díez ha sugerido que la presencia de al menos algún término teórico en las explicaciones genuinas se trata en realidad de una hipótesis metaempírica, a ser contrastada en las tareas de reconstrucciones de teorías particulares. Incluso si tuviera 
razón (lo que implicaría que los casos de ampliación conceptual no teórica ofrecidos no son explicativos o no están bien reconstruidos) quedaría en pie la pregunta acerca de por qué esta generalización a partir de las teorías conocidas no es más que una generalización accidental. ¿Por qué motivo las teorías para ser explicativas deben poseer al menos algunos conceptos cuya extensión no pueda determinarse en su prescindencia? Si tenemos razón, la teoría de Wallace, la teoría del origen común, e incluso la teoría de la selección natural darwiniana, serían casos en los que se amplía conceptualmente sin apelar a ningún concepto T-teórico. En ese caso habría que optar, o tales teorías no brindan explicaciones genuinas o el requisito de que toda explicación genuina debe ampliar conceptualmente sus aplicaciones pretendidas apelando al menos a un término teórico es demasiado fuerte. Como la segunda de las opciones no parece infinitamente más razonable, tal vez se podría reemplazar el requisito por uno más débil que sólo exija que haya ampliación conceptual (teórica, mixta o no teórica). Esto no implicaría ninguna pérdida para el enfoque explicativo de subsunción ampliativa, pues seguiría mostrándose el sentido en el que la cinemática galileana, por ejemplo, no nos parece tan explicativa como la mecánica clásica, pues, justamente, no amplía conceptualmente sus aplicaciones pretendidas.

\section{Conclusiones}

En la concepción heredada de las teorías científicas la observabilidad permitía determinar la base de contrastación de una teoría así como establecer el explanandum de la teoría. La base empírica era a su vez base de contrastación como aquello de lo que se quería dar cuenta. Los estructuralistas mostraron que no es necesario que un concepto sea observacional para que sea parte de la base de contrastación (basta con que sea T-no teórico en el sentido estructuralista, es decir, que puedan determinarse los valores sin apelar a las leyes de la teoría T). Hemos intentado mostrar que una tensión semejante puede percibirse en ciertas discusiones dentro de la concepción estructuralista: en este caso, respecto de la identificación de las aplicaciones pretendidas de una teoría con su base de contrastación.

Consideramos que, si bien éste es un punto que sólo cobra sentido y se percibe sobre el fondo que brindan las herramientas propuestas por el estructuralismo metateórico, es relevante para toda la filosofía de la ciencia por mostrar con mayor fineza matices de las relaciones entre la explicación y la predicción. 
La distinción teórico observacional, podemos decir, confundía entonces tres distinciones diferentes. Observabilidad, teoricidad (que tiene que ver con la contrastación) y explicatividad. La diferencia entre la primera y las otras dos formó parte de las críticas que condujeron al abandono de la concepción heredada. La diferencia entre las últimas dos es la propuesta de este trabajo. Pero como hemos intentado mostrar, tal distinción no implica una anomalía para el estructuralismo metateórico, aunque sí, una discusión respecto de los alcances de la T-teoricidad así como la apertura de un camino para realizar, dentro del marco estructuralista, nuevas distinciones que hasta ahora no tenían en cuenta, ni el estructuralismo, ni ninguna otra de las metateorías disponibles. ${ }^{8}$

\section{BIBLIOGRAFÍA}

Abreu, C., 2014, La propuesta de Robert K. Merton acerca de las teorías de alcance intermedio bajo el análisis de la metateoría estructuralista (tesis doctoral), Universidad de Tres de Febrero, Buenos Aires.

Achinstein, P., 1968, Concepts of Science, Johns Hopkins Press, Baltimore.

—, 1965, "The Problem of Theoretical Terms", American Philosophical Quarterly, vol. 2, no. 3, pp. 193-203.

— 1963, "Theoretical Terms and Partial Interpretation", The British Journal for the Philosophy of Science, vol. 14, no. 54, pp. 89-105.

Adams, E.W., 1955, Axiomatic Foundations of Rigid Body Mechanics (tesis doctoral), Stanford University, Stanford.

Ariza, F., 2015, Introducción de la metateoría estructuralista en la didáctica de las ciencias: didáctica modeloteórica de las ciencias (tesis doctoral), Universidad de Tres de Febrero, Buenos Aires.

Balzer, W., y C.U. Moulines (eds.), 1996, Structuralist Theory of Science, Walter de Gruiter, Berlín.

Balzer, W., C.U. Moulines y J.D. Sneed, 1987, An Architectonic for Science: The Structuralist Program, Reidel, Dordrecht. [Versión en castellano: Una arquitectónica para la ciencia, trad. Pablo Lorenzano, 2012, Universidad Nacional de Quilmes, p. 99.]

Bar-Hillel, Y., 1970, Neorealism vs. Neopositivism. A Neo-Pseudo Issue, The Magnes Press, The Hebrew University, Jerusalén.

${ }^{8}$ Queremos agradecer los comentarios de Claudio Abreu, Yefrin Ariza, Sergio Barberis, Daniel Blanco, José Díez, Adrián Ramírez y Ariel Roffé a versiones previas de este trabajo. También a los valiosos comentarios de los evaluadores anónimos. Este trabajo ha sido realizado con la ayuda de CONICET y del proyecto de investigación PICT-2012-2662 de la Agencia Nacional de Promoción Científica y Tecnológica (Argentina) [o: PICT-2012-2662 (ANPCyT, Argentina).] 
Barberis, S., 2013, La estructura de la explicación en ciencias cognitivas (tesis doctoral), Universidad de Buenos Aires, Buenos Aires.

Bartelborth, T., 2002, "Explanatory Unification”, Synthese, vol. 130, no. 1, pp. 91-107.

—_, 1996, "Scientific Explanation”, en Balzer y Moulines 1996, pp. 2343.

Blanco, D., 2012, "Primera aproximación estructuralista a la teoría del origen en común”, Ágora, vol. 31, no. 2, pp. 171-194.

Carnap, R., 1956, "The Methodological Character of Theoretical Concepts", en Feigl y Scriven 1956, pp. 38-76. [Versión en castellano: "El carácter metodológico de los conceptos teóricos", en Herbert Feigl, y Michael Scriven (eds.), 1967, Los fundamentos de la ciencia y los conceptos de la psicología y del psicoanálisis, trad. Elizabeth Grassau, Santiago, Ediciones de la Universidad de Chile, p. 53.]

Darwin, C., 1872, The Origin of Species, 6th ed., John Murray, Londres. , 1859, On the Origin of Species by Means of Natural Selection, John Murray, Londres.

Díez, J., 2014, "Scientific w-Explanation as Ampliative, Specialized Embedding: A Neo-Hempelian Account, Erkenntnis, vol. 79, no. 8, pp. 14131443 .

—_, 2012a, "Inconmensurabilidad, comparabilidad empírica y escenas observacionales", en Lorenzano y Nudler 2012b, pp. 67-118.

—— 2012b, "La explicación científica: causalidad, unificación y subsunción teórica”, en Peris-Viñé 2012, pp. 517-556.

——, 2002, "Explicación, unificación y subsunción”, en González 2002, pp. 73-93.

Díez, J., y C.U. Moulines, 1997, Fundamentos de filosofía de la ciencia, Ariel, Barcelona.

Díez, J., y P. Lorenzano (eds.), 2002, Desarrollos actuales de la metateoría estructuralista: problemas y discusiones, UNQ, Bernal.

Feigl, H. y M. Scriven (eds.), 1956, Minnesota Studies in the Philosophy of Science, vol. I: The Foundations of Science and the Concepts of Psychology and Psychoanalysis, University of Minnesota Press, Minneápolis.

Feigl, H., M. Scriven y G. Maxwell (eds.), 1958, Minnesota Studies in the Philosophy of Science, vol. 2, University of Minnesota Press, Minneápolis.

Forge, J., 2002, "Reflections on Structuralism and Scientific Explanation", Synthese, vol. 130, no. 1, pp. 109-121.

Gähde, U., 1996, "Holism and the Empirical Claim of Theory-Nets", en Balzer y Moulines 1996, pp. 167-190.

Galavotti, M., E. Nemeth y F. Stadler (eds.), 2014, European Philosophy of Science - Philosophy of Science in Europe and the Viennese Heritage, vol. 17, Springer, Dorbrecht. 
Ginnobili, S., 2012, "Reconstrucción estructuralista de la teoría de la selección natural", Ágora. Papeles de filosofía, vol. 31, no. 2, pp. 143169.

, 201la, "El estatus fenomenológico de la teoría de la selección natural", Ideas y Valores, vol. 60, no. 145, pp. 69-86.

- 2011b, La estructura de la teoría de la selección natural - Elucidación de sus conceptos fundamentales, reconstrucción de su estructura y consecuencias del análisis sobre algunas discusiones metateóricas a su alrededor (tesis doctoral), Universidad de Buenos Aires, Buenos Aires.

- 2010, "La teoría de la selección natural darwiniana", Theoria, vol. 25, no. 1, pp. 37-58.

Ginnobili, S. y D. Blanco, 2010, "Wallace y la Selección Natural (en teoría)", en Pio y Massolo 2010, pp. 251-259.

González, W. (ed.), 2002, Pluralidad de la explicación científica, Ariel, Barcelona.

Hanson, N.R., 1958, Patterns of Discovery: An Inquiry into the Conceptual Foundations of Science, Cambridge University Press, Cambridge.

Heitler, W. y F. London, 1927, "Wechselwirkung Neutraler Atome und Homöopolare Bindung nach der Quantenmechanik", Zeitschrift für Physik, vol. 44, pp. 455-472.

Hempel, C.G., 1970, “On the 'Standard Conception' of Scientific Theories”, en Radner y Winokur 1970, pp. 142-163.

Cliffs.

—_, 1958, "The Theoretician's Dilemma", en Feigl, Scriven y Maxwell 1958, pp. 37-98.

Kettlewell, H.B.D., 1956, "Further Selection Experiments on Industrial Melanism in the Lepidoptera", Heredity, vol. 10, no. 3, pp. 287-301.

- 1955, "Selection Experiments on Industrial Melanism in the Lepidoptera", Heredity, vol. 9, pp. 323-342.

Kitcher, P., 1981, "Explanatory Unification", Philosophy of Science, vol. 48, no. 4, pp. 507-531.

Kuhn, T.S., 1962, The Structure of Scientific Revolutions, University of Chicago Press, Chicago.

Lewis, D., 1970, "How to Define Theoretical Terms", The Journal of Philosophy, vol. 67, no. 13, pp. 427-446.

Lorenzano, P., 2014, "What is the Status of the Hardy-Weinberg Law within Population Genetics?", en Galavotti, Nemeth y Stadler 2014, pp. 159 172.

—— 2012, "Base empírica global de contrastación, base empírica local de contrastación y aserción empírica de una teoría", Ágora, vol. 31, no. 2, pp. 71-107.

—, 2005 , “Comentarios a 'Explicación teórica y compromisos ontológicos: un modelo estructuralista' de C.U. Moulines", Enrahonar: quaderns de filosofia, vol. 37, pp. 55-59. 
Lorenzano. P. y O. Nudler (eds.), 2012, El camino desde Kuhn. La inconmensurabilidad hoy, Biblioteca Nueva, Madrid.

Mendel, G., 1865, "Versuche über Pflanzen-Hybriden", Verhandlungen des Naturforschenden Vereins zu Brünn, vol. 4, pp. 3-57.

Merton, R.K., 1938, "Social Structure and Anomie", American Sociological Review, vol. 3, no. 5, pp. 672-682.

Moulines, C.U., 2005, “Explicación teórica y compromisos ontológicos: un modelo estructuralista", Enrahonar: quaderns de filosofia, vol. 37, pp. 37-53.

Nagel, E., P. Suppes y A. Tarski (eds.), 1962, Logic, Methodology and Philosophy of Science, Stanford University Press, Stanford.

Pauling, L., 1931, "The Nature of the Chemical Bond. Application of Results Obtained from the Quantum Mechanics and from a Theory of Paramagnetic Susceptibility to the Structure of Molecules", Journal of the American Chemical Society, vol. 53, pp. 1367-1400.

Pio, G. y A. Massolo (eds.), 2010, Epistemología e historia de la ciencia, vol. 16, Universidad Nacional de Córdoba, Córdoba.

Peris-Viñé, L.M. (ed.), 2012, Filosofía de la ciencia en Iberoamérica: metateoría estructural, Tecnos, Madrid.

Popper, K., 1962, The Logic of Scientific Discovery, Hutchinson \& Co. Ltd, Londres.

Putnam, H., 1962, "What Theories are Not", en Nagel, Suppes y Tarski 1962, pp. 240-251.

Radner, M. y S. Winokur (eds.), 1970, Minnesota Studies in the Philosophy of Science, vol. IV, University of Minnesota Press, Minneápolis.

Ruse, M., 1973, The Philosophy of Biology, Hutchinson \& Co Ltd., Londres.

Salmon, W.C., 1998, Causality and Explanation, Oxford University Press, Nueva York.

Slater, J.C., 1929, "The Theory of Complex Spectra", Physical Review, vol. 34, p. 1293.

Sneed, J.D., 1971, The Logical Structure of Mathematical Physics, Reidel, Dordrecht.

Suppe, F., 1972, "What's Wrong with the Received-View on the Structure of Scientific Theories?", Philosophy of Science, vol. 39, pp. 1-19.

Suppes, P., 1970, Set-Theoretical Structures in Science, Stanford University, Stanford.

Recibido el 27 de marzo de 2015; revisado el 20 de octubre de 2015; aceptado el 21 de enero de 2016. 